\title{
Análisis psicométrico de la Escala de Depresión Geriátrica de Yesavage en adultos mayores de la Macroregión Sur del Perú.
}

Psychometric analysis of the Yesavage Geriatric Depression Scale in elder adults from Peruvian South Macroregion.

\author{
Walter L. Arias Gallegos ${ }^{1, a, b}$, Agueda Muñoz del Carpio Toia ${ }^{2, c, d, e}$, Renzo Rivera ${ }^{3, a, f}$
}

\section{RESUMEN}

Objetivo: El presente estudio de corte psicométrico analiza la validez y confiabilidad la Escala Geriátrica de Depresión de Yesavage en una muestra 270 adultos mayores provenientes de los Centros del Adulto Mayor que funcionan en los municipios de Arequipa, Puno y Cusco. Material y métodos: Se contrastaron dos métodos de análisis factorial exploratorio uno hecho con el software SPSS 23 y otro con FACTOR 9,2 dentro del marco del proceso de validación. Resultados: Nuestros datos indican que según SPSS la escala tiene un solo factor válido y confiable con 9 ítems; mientras que FACTOR indica que la escala tiene un solo factor con 7 ítems el cual es válido; pero no confiable. Conclusiones: La Escala Geriátrica de Depresión de Yesavage presenta inconsistencias en sus propiedades psicométricas por lo que se deben hacer más estudios para determinar si debe seguir siendo utilizada en nuestro medio.

PALABRAS CLAVE: Depresión, adulto mayor, análisis factorial exploratorio, confiabilidad.

\section{SUMMARY}

Objective: The present psychometric study analyzes the validity and reliability of the Geriatric Depression Scale of Yesavage in a sample of 270 older adults from the Elderly Centers which operate in the municipalities of Arequipa, Puno, and Cusco. Materials and methods: Two exploratory factor analysis methods were contrasted, into de frame of the validation process, one made with the SPSS 23 software and another with FACTOR 9.2. Results: Our data indicate that according to SPSS the scale has valid and reliable single factor with 9 items; while FACTOR indicates the scale has a single factor with 7 items which is valid but not reliable. Conclusions: The Geriatric Depression Scale of Yesavage presents inconsistencies in its psychometric properties; consequently, more studies should be done to determine if it should continue to be used in our cities.

KEY WORDS: Depression, elder adult, exploratory factorial analysis, reliability.

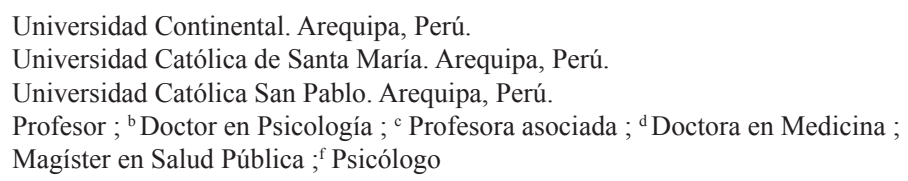




\section{INTRODUCCIÓN}

La vejez es una etapa de la vida que se caracteriza por el paulatino deterioro de la salud dependiendo del estilo de vida precedente de cada persona (1). Sin embargo, ciertas funciones psicológicas se ven afectadas con el paso del tiempo, ya que la estructura del cerebro cambia, principalmente la corteza frontal y el hipocampo (2). En tal sentido, se hace evidente la disminución de la velocidad en el procesamiento de la información y la memoria de trabajo (3). Pero estos cambios son más evidentes cuando hay procesos patológicos subyacentes que cuando el envejecimiento tiene lugar en circunstancias normales. Por ello se suele distinguir entre la senectud y la senilidad, cuando el envejecimiento es normal y cuando es patológico, respectivamente (4).

Uno de los desórdenes que más afecta a los adultos mayores, es la depresión (así como otros desórdenes del estado del ánimo), pero no es un trastorno ampliamente difundido como suele pensarse (5), ya que afecta solo al 7\% de la población gerontológica mundial (6). A pesar de ello, la depresión presenta altas tasas de comorbilidad (con la demencia principalmente (7) y se encuentra mediada por diversos factores etiológicos de carácter orgánico (8), psicológico (9) y sociocultural (10), lo que hace que amerite su evaluación y tratamiento oportunos.

En ese sentido, se han desarrollado un amplio número de instrumentos psicométricos para valorar la depresión geriátrica, entre los que se tiene la Escala de Depresión Geriátrica de Yesavage, la Escala de Depresión del Centro de Estudios Epidemiológicos, la Escala Autoaplicada para la Depresión de Zung, el Inventario de Depresión de Beck y la Escala Breve de Valoración de la Depresión con Tarjetas, entre otras (11). Aunque lo ideal es contar con marcadores biológicos que brindan información más objetiva y precisa de las emociones (12), las pruebas psicológicas cumplen una importante función de cribado que facilita la detección precoz de los desórdenes mentales o bien las situaciones de riesgo de padecerlos (6).

La Escala de Depresión Geriátrica de Yesavage fue creada en 1983 y constaba de 30 ítems orientados a valorar los síntomas de la depresión en personas mayores de 60 años (13). Luego se generó una escala abreviada de 15 ítems con niveles de confiabilidad adecuados (14), que ha sido validada en diversos países como Grecia, con un índice de consistencia interna de 0,94 y cuatro factores (15), China con índices de consistencia interna superiores a 0,7(16), España con una confiabilidad de 0,99 (17), Colombia con una estructura bifactorial e índices de consistencia interna superiores a 0,7 (18), entre otros. De hecho, se han validado incluso versiones más cortas de hasta cinco ítems, que cuentan con una consistencia interna de 0,73 y una estructura unidimensional, mientras que la escala de 15 ítems cuenta con una confiabilidad mayor y dos factores que confirman su validez de constructo (19).

Una salvedad que merece mayor precisión es que varios de estos estudios han utilizado el programa SPSS para procesar los datos y obtener la validez de constructo $(18,19)$, lo que supone un análisis de variables continuas a través de matrices de correlación de Pearson. Sin embargo, la Escala de Depresión Geriátrica de Yesavage tiene una escala de respuesta dicotómica (verdadero/falso) que implica que, la estructura factorial depende más del grado de dificultad que del constructo subyacente (20). En tal sentido, es más recomendable utilizar correlaciones tetracóricas que producto-momento de Pearson como utiliza, por defecto, el SPSS (Sattistical Package for Social Sciences) al efectuar el análisis factorial, mientras que otros programas como FACTOR sí permiten analizar diferentes instrumentos de medición dependiendo de su tipo de respuesta (21).

Por ello, el presente trabajo de investigación, pretende hacer un análisis psicométrico de la Escala de Depresión Geriátrica de Yesavage en adultos mayores de la Macroregión sur del Perú, que incluye tres regiones principalmente. Asimismo, se trabajará con el software SPSS y el programa Factor, que suele ser más exhaustivo que el programa SPSS con el que la mayoría de los estudios mencionados se han llevado a cabo $(18,19)$. En consecuencia, nuestro objetivo es valorar la validez de constructo y la confiabilidad de dicho instrumento, contrastando el procesamiento psicométrico mediante el programa SPSS y el programa FACTOR.

\section{MATERIAL Y MÉTODOS}

El estudio es de tipo instrumental (22), pues se trata de valorar las propiedades psicométricas de la Escala de Depresión Geriátrica de Yesavage. Asimismo, la muestra fue seleccionada de manera no probabilística mediante la técnica de grupos intactos de los Centros del Adulto Mayor que funcionan en los municipios de estas ciudades. 
Se empleó la Escala de Depresión Geriátrica de Yesavage de 30 ítems (13), que fue adaptada al español por Izal et al., (23), tiene una escala de respuesta dicotómica, de modo que un puntaje de 0 a 10 indica que no hay indicios de depresión, pero puntajes por encima de 10 indican que la persona atraviesa por un estado depresivo, que requiere de una evaluación clínica exhaustiva para confirmar el diagnóstico y brindar el tratamiento pertinente.

Por otro lado, la muestra fue evaluada en los Centros del adulto Mayor de las regiones de Arequipa y Puno, a quienes se les administró la escala luego de haber llenado el consentimiento informado previa explicación de los fines del estudio dar la garantía de la reserva y confidencialidad de los datos. El Comité de Ética de la Universidad Católica de Santa María aprobó la realización del presente estudio que forma parte de una investigación mayor sobre diversas variables que impactan la salud de los adultos mayores de la Macroregión Sur, y que serán objeto de análisis en otras publicaciones.

Una vez recogida la información, se procedió a procesar los datos con el programa SPSS (versión 23) (24) y el programa FACTOR (versión 9.2)(25). El procesamiento de los datos implicó el análisis descriptivo de los ítems (para ambos tipos de

Tabla 1. Estadísticos descriptivos obtenidos mediante procesamiento con SPSS

\begin{tabular}{|c|c|c|c|c|c|c|c|}
\hline $\mathbf{N}^{\circ}$ & Mín. & Máx. & Media & D.E. & Asimetría & Curtosis & citc \\
\hline Ítem 1 & 0 & 1 & 0,570 & 0,495 & $-0,286$ & $-1,933$ & 0,471 \\
\hline Ítem 2 & 0 & 1 & 0,737 & 0,441 & $-1,083$ & $-0,834$ & 0,353 \\
\hline Ítem 3 & 0 & 1 & 0,692 & 0,462 & $-0,839$ & $-1,305$ & 0,356 \\
\hline Ítem 4 & 0 & 1 & 0,722 & 0,448 & $-0,998$ & $-1,012$ & 0,347 \\
\hline Ítem 5 & 0 & 1 & 0,648 & 0,478 & $-0,624$ & $-1,623$ & 0,088 \\
\hline Ítem 6 & 0 & 1 & 0,766 & 0,423 & $-1,268$ & $-0,395$ & 0,606 \\
\hline Ítem 7 & 0 & 1 & 0,392 & 0,489 & 0,442 & $-1,818$ & 0,373 \\
\hline Ítem 8 & 0 & 1 & 0,581 & 0,494 & $-0,332$ & $-1,904$ & 0,544 \\
\hline Ítem 9 & 0 & 1 & 0,500 & 0,500 & 0,000 & $-2,015$ & 0,481 \\
\hline Ítem 10 & 0 & 1 & 0,755 & 0,430 & $-1,196$ & $-0,574$ & 0,503 \\
\hline Ítem 11 & 0 & 1 & 0,722 & 0,448 & $-0,998$ & $-1,012$ & 0,197 \\
\hline Ítem 12 & 0 & 1 & 0,488 & 0,500 & 0,045 & $-2,013$ & 0,356 \\
\hline Ítem 13 & 0 & 1 & 0,818 & 0,386 & $-1,662$ & 0,768 & 0,211 \\
\hline Ítem 14 & 0 & 1 & 0,518 & 0,500 & $-0,075$ & $-2,009$ & 0,399 \\
\hline Ítem 15 & 0 & 1 & 0,259 & 0,439 & 1,105 & $-0,785$ & 0,375 \\
\hline Ítem 16 & 0 & 1 & 0,666 & 0,472 & $-0,711$ & $-1,506$ & 0,576 \\
\hline Ítem 17 & 0 & 1 & 0,596 & 0,491 & $-0,395$ & $-1,858$ & 0,543 \\
\hline Ítem 18 & 0 & 1 & 0,548 & 0,498 & $-0,195$ & $-1,977$ & 0,326 \\
\hline Ítem 19 & 0 & 1 & 0,366 & 0,482 & 0,556 & $-1,703$ & 0,204 \\
\hline Ítem 20 & 0 & 1 & 0,670 & 0,470 & $-0,729$ & $-1,480$ & 0,126 \\
\hline Ítem 21 & 0 & 1 & 0,451 & 0,498 & 0,195 & $-1,977$ & 0,398 \\
\hline Ítem 22 & 0 & 1 & 0,455 & 0,498 & 0,179 & $-1,983$ & 0,508 \\
\hline Ítem 23 & 0 & 1 & 0,474 & 0,500 & 0,104 & $-2,004$ & 0,351 \\
\hline Ítem 24 & 0 & 1 & 0,533 & 0,499 & $-0,134$ & $-1,997$ & 0,350 \\
\hline Ítem 25 & 0 & 1 & 0,640 & 0,480 & $-0,590$ & $-1,664$ & 0,357 \\
\hline Ítem 26 & 0 & 1 & 0,681 & 0,466 & $-0,783$ & $-1,397$ & 0,547 \\
\hline Ítem 27 & 0 & 1 & 0,288 & 0,454 & 0,937 & $-1,131$ & 0,513 \\
\hline Ítem 28 & 0 & 1 & 0,529 & 0,500 & $-0,119$ & $-2,001$ & 0,358 \\
\hline Ítem 29 & 0 & 1 & 0,451 & 0,498 & 0,195 & $-1,977$ & $-0,097$ \\
\hline Ítem 30 & 0 & 1 & 0,692 & 0,462 & $-0,839$ & $-1,305$ & 0,140 \\
\hline
\end{tabular}


Tabla 2. Matriz de estructura obtenida mediante el método de Máxima verosimilitud con SPSS

\begin{tabular}{|c|c|c|c|c|c|c|c|}
\hline \multirow{2}{*}{ Ítems } & \multicolumn{7}{|c|}{ Factores } \\
\hline & 1 & 2 & 3 & 4 & 5 & 6 & 7 \\
\hline Ítem 1 & 0.372 & & & & & & \\
\hline Ítem 2 & & & & 0.450 & & & \\
\hline Ítem 3 & & & & & & & 0.321 \\
\hline Ítem 4 & & 0.496 & & & & & \\
\hline Ítem 6 & 0.999 & & & & & & \\
\hline Ítem 7 & & & 0.319 & & & & \\
\hline Ítem 8 & & 0.532 & & & & & \\
\hline Ítem 9 & & & 0.393 & & & & \\
\hline Ítem 10 & & & & 0.431 & & & \\
\hline Ítem 12 & & & & & 0.287 & & \\
\hline Ítem 13 & & 0.415 & & & & & \\
\hline Ítem 14 & & & & & 0.479 & 0.224 & \\
\hline Ítem 15 & & & 0.580 & & & & \\
\hline Ítem 16 & & & 0.453 & & & & \\
\hline Ítem 17 & 0.422 & & & & & & \\
\hline Ítem 18 & & 0.483 & & & & & \\
\hline Ítem 19 & 0.383 & & & & & & \\
\hline Ítem 21 & 0.379 & & & & & & \\
\hline Ítem 22 & & & & & 0.380 & & \\
\hline Ítem 23 & & 0.411 & & & & & \\
\hline Ítem 24 & & 0.492 & & & & & \\
\hline Ítem 25 & & 0.652 & & & & & \\
\hline Ítem 26 & & 0.457 & & & & & \\
\hline Ítem 27 & & 0.430 & & & & 0.226 & \\
\hline Ítem 28 & 0.306 & & & & & & \\
\hline Varianza explicada & $11.4 \%$ & $11.64 \%$ & $7.16 \%$ & $5.59 \%$ & $5.1 \%$ & $4.01 \%$ & $3.27 \%$ \\
\hline
\end{tabular}

procesamiento) y el análisis factorial para determinar la validez de constructo (mediante el método de máxima similitud con el SPSS y con el método de mínimos cuadrados no ponderados robustos), así como el análisis de la consistencia interna para determinar la confiabilidad (con la prueba alfa de Cronbach para ambos tipos de procesamiento y la Omega de McDonald solo con el FACTOR).

\section{RESULTADOS}

La muestra está constituida por 270 adultos mayores con una edad media de 73 años y una desviación estándar de $\pm 6,3$ dentro de un rango de 60 a 92 años, de los cuales el $34,8 \%$ son varones y el $65,2 \%$ son mujeres. En cuanto a su lugar de procedencia, el $58,9 \%$ son de Arequipa, el 33,7\% de Puno y el 7,4\% de Cusco, ciudades ubicadas en la Macroregión Sur del Perú. En la tabla 1 se tienen los estadísticos descriptivos obtenidos mediante el programa SPSS donde se puede apreciar que los promedios de respuesta por ítem van de 0,259 para el ítem 15 (“¿Piensa que es maravilloso vivir?”) hasta 0,818 para el ítem 13 (“¿Frecuentemente está preocupado por el futuro?”). Asimismo, las medidas de asimetría son bajas pues ningún valor se ubica dentro del rango $-2 /+2$, sin embargo, las curtosis obtienen valores fuera de este rango en los ítems 9, 12, 14, 23,28. También se pueden apreciar las correlaciones ítem test calculadas con la correlación producto momento de Pearson que tienen puntuaciones superiores a 0,2 , excepto en los ítems 5, 11, 20, 29 y 30; motivo por el cual tuvieron que ser eliminados. 
Tabla 3. Matriz de estructura final y sus niveles de confiabilidad con SPSS

\begin{tabular}{lrrrrr}
\hline & \multicolumn{1}{l}{ F1 } & \multicolumn{1}{l}{ F2 } & \multicolumn{1}{l}{ F3 } & \multicolumn{1}{c}{ F4 } & \multicolumn{1}{c}{ Total } \\
\hline Media & 3.281 & 5.288 & 1.818 & 1.463 & 11.851 \\
Mediana & 4.000 & 5.500 & 2.000 & 1.000 & 12.500 \\
Moda & 4.00 & 8.00 & 2.00 & 1.00 & 18.00 \\
Desv. Desviación & 1.733 & 2.452 & 1.243 & 1.036 & 4.655 \\
Varianza & 3.006 & 6.013 & 1.547 & 1.075 & 21.673 \\
Asimetría & -0.522 & -0.394 & 0.068 & 0.170 & -0.430 \\
Curtosis & -0.549 & -0.705 & -1.038 & -1.135 & -0.690 \\
Rango & 6.00 & 9.00 & 4.00 & 3.00 & 21.00 \\
Mínimo & 0.00 & 0.00 & 0.00 & 0.00 & 1.00 \\
Máximo & 6.00 & 9.00 & 4.00 & 3.00 & 22.00 \\
$\alpha$ & 0.642 & 0.751 & 0.552 & 0.453 & 0.803 \\
F1 & 1 & 0.218 & 0.586 & 0.337 & 0.719 \\
F2 & & 1 & 0.221 & 0.421 & 0.761 \\
F3 & & & 1 & 0.371 & 0.684 \\
F4 & & & & 1 & 0.669 \\
Total & & & & & 1 \\
\hline
\end{tabular}

Seguidamente, se procedió a valorar la validez de constructo de la Escala de Depresión Geriátrica de Yesavage, para lo cual se realizó un análisis factorial exploratorio con el método de máxima verosimilitud y rotación Promím. Los resultados arrojaron un valor bajo de adecuación muestral, a través de la prueba KaiserMeyer-Olkin (KMO; 0,697) aunque significativo en la prueba de esfericidad de Bartlett $\left(\mathrm{x}^{2}=2319,885\right.$; $\mathrm{gl}=$ $300 ; \mathrm{p}=0,000)$. Del análisis factorial practicado se obtuvieron siete factores que explican el $52,7 \%$ de la varianza total de la prueba. En el primer factor saturan los ítems 1, 6, 17, 19, 21 y 28; y explicó el $11,4 \%$ de la varianza, mientras que en el segundo factor saturan los ítems 4, 8, 13, 18, 23, 24, 25, 26 y 27 y explicó el $11.64 \%$ de la varianza de la prueba. En el tercer factor saturaron los ítems 7, 9, 15 y 16 explicando el 7,16\% de la varianza, el cuarto factor se compone los ítems 2 y 10 explicando el 5,59\% de la varianza, el quinto factor comprende los ítems 12,14 y 22 con el $5,1 \%$ de la varianza explicada, y el séptimo factor se compone del ítem 3 que explica el 3,27\% de la varianza. Los ítems del sexto factor tienen un bajo índice de saturación que explicaba el $4,01 \%$ de la varianza total (tabla 2).

Dados estos valores se tuvieron que eliminar los factores 4, 6 y 7, porque solo una escasa cantidad de ítems (menos de tres) saturaron en ellos. En la tabla 3 se tienen los valores descriptivos de los factores restantes, así como las correlaciones interfactoriales que en su mayoría tienen coeficientes de correlación superiores a 0,3. También se tienen los valores de la confiabilidad obtenida mediante el método de consistencia interna y la prueba alfa de Cronbach. En ese sentido, solo el segundo factor obtuvo un índice adecuado $(\alpha=0,751)$, por lo que los factores restantes no son confiables. Puede decirse entonces, que a través del SPSS, la Escala de Depresión Geriátrica de Yesavage, no cuenta con índices de validez y confiabilidad aceptables, y que básicamente, solo los ítems 4, 8, 13, 18, 23, 24, 25, 26 y 27 que comprenden el segundo factor y explican el $11,64 \%$ de la varianza total de la prueba, son confiables y se relacionan con la labilidad emocional y la desesperanza propia de la depresión.

Ahora bien, con el programa FACTOR se realizó un procedimiento similar pero más robusto, pues las matrices de correlación fueron de tipo tetracórico y se trabajó con el procedimiento de óptima implementación de análisis paralelo en vez de la regla de Kaiser. En la tabla 4 se pueden apreciar los estadísticos descriptivos tales como la media, los intervalos de confianza, la varianza, la asimetría y la curtosis. Mediante este procesamiento los datos de la asimetría y la curtosis obtienen mejores valores que con el procesamiento mediante SPSS, ya que se ubican dentro del rango de $-1 /+1$.

Por otro lado, al realizar el análisis factorial, se empleó el método de mínimos cuadrados no ponderados robustos, obteniéndose también un índice 
Tabla 4. Estadísticos descriptivos obtenidos mediante procesamiento con FACTOR

\begin{tabular}{lccccc}
\hline No & Media & $\begin{array}{c}\text { Intervalo de } \\
\text { confianza }\end{array}$ & Varianza & Asimetría & Curtosis \\
\hline Ítem 1 & 0.570 & $(0.49 ; 0.65)$ & 0.245 & -0.285 & -1.915 \\
Ítem 2 & 0.737 & $(0.67 ; 0.81)$ & 0.194 & -1.081 & -0.832 \\
Ítem 3 & 0.693 & $(0.62 ; 0.76)$ & 0.213 & -0.838 & -1.297 \\
Ítem 4 & 0.722 & $(0.65 ; 0.79)$ & 0.201 & -0.996 & -1.008 \\
Ítem 5 & 0.648 & $(0.57 ; 0.72)$ & 0.228 & -0.623 & -1.610 \\
Ítem 6 & 0.767 & $(0.70 ; 0.83)$ & 0.179 & -1.266 & -0.400 \\
Ítem 7 & 0.393 & $(0.32 ; 0.47)$ & 0.238 & 0.442 & -1.802 \\
Ítem 8 & 0.581 & $(0.50 ; 0.66)$ & 0.243 & -0.332 & -1.887 \\
Ítem 9 & 0.500 & $(0.42 ; 0.58)$ & 0.250 & 0.000 & -1.996 \\
Ítem 10 & 0.756 & $(0.69 ; 0.82)$ & 0.185 & -1.194 & -0.577 \\
Ítem 11 & 0.722 & $(0.65 ; 0.79)$ & 0.201 & -0.996 & -1.008 \\
Ítem 12 & 0.489 & $(0.41 ; 0.57)$ & 0.250 & 0.045 & -1.994 \\
Ítem 13 & 0.819 & $(0.76 ; 0.88)$ & 0.149 & -1.659 & 0.746 \\
Ítem 14 & 0.519 & $(0.44 ; 0.60)$ & 0.250 & -0.074 & -1.991 \\
Ítem 15 & 0.259 & $(0.19 ; 0.33)$ & 0.192 & 1.103 & -0.785 \\
Ítem 16 & 0.667 & $(0.59 ; 0.74)$ & 0.222 & -0.710 & -1.494 \\
Ítem 17 & 0.596 & $(0.52 ; 0.67)$ & 0.241 & -0.394 & -1.842 \\
Ítem 18 & 0.548 & $(0.47 ; 0.63)$ & 0.248 & -0.194 & -1.959 \\
Ítem 19 & 0.367 & $(0.29 ; 0.44)$ & 0.232 & 0.555 & -1.689 \\
Ítem 20 & 0.670 & $(0.60 ; 0.74)$ & 0.221 & -0.728 & -1.469 \\
Ítem 21 & 0.452 & $(0.37 ; 0.53)$ & 0.248 & 0.194 & -1.959 \\
Ítem 22 & 0.456 & $(0.38 ; 0.53)$ & 0.248 & 0.179 & -1.964 \\
Ítem 23 & 0.474 & $(0.40 ; 0.55)$ & 0.249 & 0.104 & -1.985 \\
Ítem 24 & 0.533 & $(0.46 ; 0.61)$ & 0.249 & -0.134 & -1.978 \\
Ítem 25 & 0.641 & $(0.57 ; 0.72)$ & 0.230 & -0.589 & -1.651 \\
Ítem 26 & 0.681 & $(0.61 ; 0.75)$ & 0.217 & -0.782 & -1.387 \\
Ítem 27 & 0.289 & $(0.22 ; 0.36)$ & 0.205 & 0.935 & -1.125 \\
Ítem 28 & 0.530 & $(0.45 ; 0.61)$ & 0.249 & -0.119 & -1.982 \\
Ítem 29 & 0.452 & $(0.37 ; 0.53)$ & 0.248 & 0.194 & -1.959 \\
Ítem 30 & 0.693 & $(0.62 ; 0.76)$ & 0.213 & -0.838 & -1.297 \\
\hline & & & & & \\
\hline
\end{tabular}

de adecuación muestral bajo, mediante la prueba $\operatorname{KMO}(0,654)$ y una estructura unidimensional que explica el $10,46 \%$ de la varianza total de la prueba. En ese sentido, solo los ítems 6, 8, 16, 17, 21, 25 y 26 obtuvieron saturaciones superiores a 0.3 en el factor hallado. Por otro lado, la prueba de congruencia unidimensional (I-UNICO) la varianza común explicada por ítem (I-ECV) y las cargas absolutas residuales de cada ítem (I-REAL) no son concluyentes en que la escala sea unidimensional, como se puede apreciar en la tabla 5 .
Asimismo, solo dos de cinco índices de bondad de ajuste satisfacen los criterios establecidos para la confirmación de un modelo factorial adecuado $\left(\chi^{2}=668,078 ; \mathrm{gl}=405(p<, 000) ; \chi^{2} / \mathrm{gl}=1,649 ; \mathrm{CFI}=\right.$ 0,553 IC $95 \%$ [0,423, 56,697]; GFI $=0,876$ IC 95\% $[0,837,0,944] ;$ AGFI $=0,867$ IC 95\% [0,825, 0,940]; RMSEA $=0,049$ IC $95 \%[0,005 ; 0,067])$, lo que pone en duda la adecuación del modelo factorial. Finalmente, a pesar que solo siete ítems obtuvieron saturaciones adecuadas, el cálculo la confiabilidad mediante la prueba alfa de Cronbach, arrojó un índice de 0,5 , mientras que la prueba Omega de McDonald 
Tabla 5. Matriz de estructura y evaluación de unidimensionalidad con FACTOR

\begin{tabular}{lccccc}
\hline $\mathbf{N}^{\circ}$ & Factor 1 & Comunalidad & I-UNICO & I-ECV & I-REAL \\
\hline Ítem 1 & -0.231 & 0.053 & 0.934 & 0.723 & 0.176 \\
Ítem 2 & -0.044 & 0.002 & 0.505 & 0.369 & 0.069 \\
Ítem 3 & -0.029 & 0.001 & 0.440 & 0.329 & 0.053 \\
Ítem 4 & -0.080 & 0.006 & 0.438 & 0.327 & 0.140 \\
Ítem 5 & 0.020 & 0.000 & 0.066 & 0.062 & 0.106 \\
Ítem 6 & -0.872 & 0.760 & 0.988 & 0.864 & 0.320 \\
Ítem 7 & -0.000 & 0.000 & 0.000 & 0.000 & 0.001 \\
Ítem 8 & -0.315 & 0.099 & 0.952 & 0.757 & 0.184 \\
Ítem 9 & -0.230 & 0.053 & 0.938 & 0.730 & 0.154 \\
Ítem 10 & -0.173 & 0.030 & 1.000 & 0.977 & 0.035 \\
Ítem 11 & -0.010 & 0.000 & 0.387 & 0.296 & 0.017 \\
Ítem 12 & -0.056 & 0.003 & 0.207 & 0.175 & 0.139 \\
Ítem 13 & -0.001 & 0.000 & 0.000 & 0.000 & 0.267 \\
Ítem 14 & -0.049 & 0.002 & 0.667 & 0.472 & 0.060 \\
Ítem 15 & -0.128 & 0.016 & 0.488 & 0.359 & 0.262 \\
Ítem 16 & -0.437 & 0.191 & 0.944 & 0.740 & 0.331 \\
Ítem 17 & -0.321 & 0.103 & 0.997 & 0.928 & 0.114 \\
Ítem 18 & -0.094 & 0.009 & 0.140 & 0.124 & 0.335 \\
Ítem 19 & -0.263 & 0.069 & 0.591 & 0.423 & 0.346 \\
Ítem 20 & -0.001 & 0.000 & 0.221 & 0.184 & 0.002 \\
Ítem 21 & -0.311 & 0.097 & 0.780 & 0.555 & 0.359 \\
Ítem 22 & -0.208 & 0.043 & 0.938 & 0.731 & 0.164 \\
Ítem 23 & -0.022 & 0.000 & 0.036 & 0.034 & 0.151 \\
Ítem 24 & -0.143 & 0.020 & 0.240 & 0.198 & 0.435 \\
Ítem 25 & -0.429 & 0.184 & 0.687 & 0.486 & 0.597 \\
Ítem 26 & -0.328 & 0.107 & 0.610 & 0.435 & 0.505 \\
Ítem 27 & -0.238 & 0.057 & 0.998 & 0.939 & 0.091 \\
Ítem 28 & -0.083 & 0.007 & 0.729 & 0.516 & 0.097 \\
Ítem 29 & 0.001 & 0.000 & 0.016 & 0.016 & 0.007 \\
Ítem 30 & -0.011 & 0.000 & 0.251 & 0.206 & 0.024 \\
\hline & & & & &
\end{tabular}

obtuvo un índice de consistencia interna aún menor $(0,453)$, esto sugiere que la prueba no es confiable.

\section{DISCUSIÓN}

La depresión es un trastorno que amerita ser atendido (26) y diferenciado de otros múltiples desórdenes emocionales (27), sobre todo entre la población adulta mayor, que al percibir que su salud y sus facultades declinan con el tiempo, suelen deprimirse. Además, en este grupo etario la depresión se solapa muy frecuentemente con la demencia (7), por lo que es necesario contar con instrumentos de evaluación debidamente validados. La finalidad del presente estudio ha sido la valoración de las cualidades psicométricas de la Escala de Depresión Geriátrica de Yesavage, que como su nombre indica, es usada para evaluar la depresión en los adultos mayores.

Nuestro estudio ha permitido calcular la validez y la confiabilidad de la Escala de Depresión Geriátrica de Yesavage en adultos mayores de entre 60 y 92 años, provenientes de provincias ubicadas en la Macro región sur del Perú. Los resultados, sin embargo, no respaldan que se trate de un instrumento válido ni confiable. En ese sentido, aunque otros estudios en países de habla hispana, han dado cuenta de las bondades psicométricas de este instrumento (17), 
han empleado el programa SPSS en la realización del análisis factorial, reportado una estructura interna de dos, tres o cuatro factores con buenos índices de confiabilidad en diversas partes del mundo (18), muchos de ellos han utilizado el programa SPSS para realizar los cálculos psicométricos, pero por defecto este programa utiliza las correlaciones de Pearson para efectuar el análisis factorial, cuando los más conveniente, dado el tipo de respuesta dicotómica que ofrece la prueba, es que se realicen correlaciones tetracóricas (20).

Para nuestro caso, el análisis con el SPSS ha reportado una estructura de siete factores que se reduce a solo cuatro después de eliminar los factores que se componen de menos de tres ítems. Estos cuatro factores se reducen a solo uno, considerando la baja confiabilidad que han obtenido los tres restantes que terminaron siendo eliminados. De modo que, mediante el procesamiento con SPSS la estructura factorial de la Escala de Depresión Geriátrica de Yesavage es unidimensional y cuenta con una confiabilidad aceptable $(0,751)$. Los ítems que la componen serían el $4,8,13,18,23,24,25,26$ y 27 , que explican el $11,64 \%$ de la varianza total de la prueba, es decir, tienen un escaso poder predictivo, pues lo ideal es que la varianza explicada supere el 40\% (28). En tal sentido, otros estudios, como el de Gómez-Angulo y Campo-Arias (19) también han reportado que una versión breve de incluso 5 ítems es más confiable que la versión de 15 ítems.

Por otro lado, al procesar los datos con el programa FACTOR la confiabilidad obtenida es baja $(<0.6)$ y la estructura interna unidimensional, que comprende los ítems $6,8,16,17,21,25$ y 26 con saturaciones por encima de 0,3 y valores que confirman la unidimensionalidad de la prueba, pero con bajos índices de bondad de ajuste. Es decir, la valoración psicométrica de la Escala de Depresión Geriátrica de Yesavage se compone de siete ítems, no tiene una adecuada solución factorial y es poco confiable. Estos resultados, son empero, más favorables con el programa SPSS, pero como se ha venido explicando los criterios de factorización son menos robustos.

Los resultados obtenidos demuestran que no es conveniente realizar estimaciones psicométricas de escalas dicotómicas con el programa SPSS, ya que se violan ciertos supuestos psicométricos que favorecen los resultados de los cálculos estadísticos (21). Esto tendría como consecuencia, una falsa valoración de las funciones psicológicas de los adultos mayores, y por consiguiente, una errada toma de decisiones en cuanto a su diagnóstico y tratamiento. En ese sentido, dos situaciones podrían explicar nuestros resultados: o bien la prueba no tiene propiedades psicométricas adecuadas que satisfaga criterios estadísticos robustos, o bien la muestra no ha respondido adecuadamente a los ítems del instrumento. En ambos casos, estos resultados pueden estar mediados por diferencias culturales y los niveles socioeconómicos de donde provienen los adultos mayores evaluados. En consecuencia, nuevas investigaciones deberán dar cuenta de las propiedades psicométricas de la Escala de Depresión Geriátrica de Yesavage, pero lo ideal es que se efectúen con técnicas psicométricas más rigurosas, tales como análisis factorial confirmatorio, análisis de invarianza, teoría de respuesta al ítem, etc.

Por otro lado, los ítems 8 ( $¿$ Tiene miedo de que algo le esté pasando?), 25 (¿Frecuentemente siente ganas de llorar?) y 26 (¿Tiene problemas para concentrarse?) obtienen adecuadas medidas en ambos procedimientos, y podrían estar haciendo referencia a una problemática común en el adulto mayor de la Macroregión sur del Perú, como es el miedo a padecer alguna enfermedad, labilidad emocional y un decremento de las funciones psicológicas superiores. En ese sentido, así se valoran aspectos negativos de la vida afectiva del adulto mayor, también debe promoverse el estudio de su bienestar psicológico, que suele estar mediado por el apoyo percibido, los estilos de afrontamiento, la satisfacción con la vida (29) y la resiliencia (30), así como variables familiares y psicosociales (31).

\section{Correspondencia}

\author{
Walter L. Arias Gallegos \\ Universidad Continental, \\ Av. Los Incas S/N, José Luis Bustamante y Rivero, \\ Arequipa, Perú. \\ Correo electrónico: warias@continental.edu.pe
}

\section{REFERENCIAS BIBLIOGRAFICAS}

1. Papalia DE, Sterns HL, Feldman RD, Camp, C. J. Desarrollo del adulto y vejez. Ciudad de México: McGraw-Hill; 2009.

2. Alemán A. Cuando el cerebro envejece. Bogotá: Ícono; 2016.

3. Vásquez J, Morante P, Soto M. Velocidad de procesamiento y memoria de trabajo en adultos mayores: implicancias para el envejecimiento normal y patológico. Revista de Psicología. 2011; 1(1): 1325.

4. Craig G. Desarrollo psicológico. Ciudad de México: 
Prentice Hall; 2009.

5. Stucchi-Portocarrero S. ¿Realmente existe una "epidemia de depresión"? Rev Neuropsiquiatr. 2017; 80(4): 261-264.

6. Riquelme A, Buendía J, López AI. Desarrollo y validación de un instrumento para la evaluación de la depresión en ancianos. Psicothema. 2016; 18(2): 28892.

7. Peña J, Tardón A, Menéndez IA, Blázquez B. Prevalencia de demencia en población mayor de 80 años. Psicothema. 2000; 12(2): 431-7.

8. Carnés-Vendrell A, Deus-Yela J, Molina-Seguin J, Pifarré-Paradero J, Purroy F. Actualización de la depresión postictus: nuevos retos en pacientes con ictus minor o ataque isquémico transitorio. Rev Neurol. 2016; 62(10): 460-7.

9. Ballón ML, Montesinos LC. Inteligencia emocional en adultos mayores de asilos públicos e instituciones privadas de Arequipa. Revista de Psicología de Arequipa. 2012; 2(1): 49-63.

10. Salaberría K, Polo-López R, Cruz-Sáez S, Echeburúa, E. Chronic stress immigrants and relatives of people with mental illness: a comparative study. Revista Mexicana de Psicología. 2015; 32(1): 7-15.

11. Cabañero-Martínez MJ, Cabrero-García J, RichartMartínez, M, Muñoz-Mendoza CL, Reig-Ferrer A. Revisión estructurada de las escalas de depresión en personas mayores. Int J Clin Health Psychol. 2007; 7(3): 823-46.

12. Garzón-Rey J, Arza A, de la Cámara C, Lobo A, Armario A, Aguiló J. Aproximación a una escala de referencia de estrés emocional agudo. Rev Neurol. 2017; 64(12): 529-37.

13. Yesavage JA, Brink TL, Rose TL, Lum O, Huang V, Adey M, Leiter VO. Development and validation of a geriatric depression screening scale: a preliminary report. J Psychiat Res. 1983; 17: 37-49.

14. Sheikh JL, Yesavage JA. Geriatric depression Scale (GDS): Recent evidence and development of a shorter version. Clin Geront. 1986; 5: 165-173.

15. Fountoulakis KN, Tsolaki M, Yesavage J, Ohara R, Kazis A, Validation of a short from of the Geriatric Depression Scale (GDS) in Greece. Aging Clin Exp Res. 1999; 11: 367-72.

16. Chen ST, Chan ACM. A brief version of the Geriatric depression Scale for the Chinese. Psychol Asess. 2004; 16: 182-6.

17. Martínez J, Onís MO, Dueñas R, Albert C, Aguado C, Luque R. Versión española del cuestionario de Yesavage abreviado (GDS), para el despistaje de depresión en mayores de 65 años: adaptación y validación. MEDIFAM. 2002; 12: 620-30.

18. Campo-Arias A, Urruchurtu Y, Solano T, Vergara AJ, Cogollo Z. Consistencia interna, estructura factorial y confiabilidad del constructo de la escala de Yesavage para depresión geriátrica (GDS-15) en Cartagena
(Colombia). Salud Uninorte Barranquilla (Col.). 2008; 24(1): 1-9.

19. Gómez-Angulo C, Campo-Arias A. Escala de Yesavage para Depresión Geriátrica (GDS-15 y GDS-5): estudio de la consistencia interna y estructura factorial. Univ Psychol. 2011; 10(3): 735-43.

20. Dominguez SA. ¿Matrices policóricas/tetracóricas o matrices Pearson? Un estudio metodológico. Revista Argentina de Ciencias del Comportamiento. 2014; 6(1): 39-48.

21. Freiberg A, Stover JB, dela Iglesia G, Fernández M. Correlaciones policóricas y tetracóricas en estudios factoriales exploratorios y confirmatorios. Ciencias Psicológicas. 2013; 7(2): 151-64.

22. Montero I, León OG. A guide for naming research studies in Psychology. Int J Clin Health Psychol. 2007; 7(3): 847-62.

23. Izal M, Montorio I. Adaptación en nuestro medio de la Escala de Depresión Geriátrica (GDS) en distintos subgrupos: residentes en la comunidad y asistentes a hospitales de día. Rev Gerontol. 1996; 6: 329-37.

24. International Business Machines Corporation. IBM SPSS Statistics (Version 20) [programa informático en CD-ROM]. New York: International Business Machines Corporation; 2011.

25. Lorenzo-Seva U, Ferrando PJ. Factor 9.2 A comprehensive program for fitting exploratory and semiconfirmatory factor analysis and IRT models. Applied Psychological Measurement. 2013; 37(6): 497-8.

26. González S, Fernández C, Pérez J, Amigo I. Prevención secundaria de la depresión en atención primaria. Psicothema. 2006; 18(3): 471-7.

27. García J, Fernández-Ballesteros R, Montero I, Heiby EM. Multiple correlates of unipolar depression: contributions from the paradigmatic behavioral theory. Psicothema. 1995; 7(1): 41-50.

28. Burga A. La unidimensionalidad de un instrumento de medición: perspectiva factorial. Rev Psicol (Lima). 2006; 24(1): 53-80.

29. Torres WI, Flores MM. Factores predictores del bienestar subjetivo en adultos mayores. Rev psicol (Lima). 2018; 63(1): 9-48.

30. Caycho-Rodríguez T, Ventura-León J, GarcíaCadena CH, Tomás JM, Domínguez-Vergara J, Daniel L, Arias-Gallegos WL. Evidencias psicométricas de una medida breve de resiliencia en adultos mayores no institucionalizados. Psychosocial Intervention. 2018; 27: 73-9.

31. Arias WL, Yépez L, Núñez AL, Oblitas A, Pinedo S, Masías MA, Hurtado J. Felicidad, depresión y creencia en la benevolencia humana en adultos mayores institucionalizados y no institucionalizados. Propósitos y Representaciones. 2013; 1(2): 83-103. 